2001

\title{
Self-Consistent Simulations of Electroporation Dynamics in Biological Cells Subjected to Ultrashort Electrical Pulses
}

\author{
R. P. Joshi \\ Old Dominion University \\ Q. $\mathrm{Hu}$ \\ Old Dominion University \\ R. Aly \\ Old Dominion University \\ K.H. Schoenbach \\ Old Dominion University \\ H. P. Hjalmarson
}

Follow this and additional works at: https://digitalcommons.odu.edu/bioelectrics_pubs

Part of the Cell Biology Commons, and the Electrical and Computer Engineering Commons

\section{Repository Citation}

Joshi, R. P.; Hu, Q.; Aly, R.; Schoenbach, K. H.; and Hjalmarson, H. P., "Self-Consistent Simulations of Electroporation Dynamics in Biological Cells Subjected to Ultrashort Electrical Pulses" (2001). Bioelectrics Publications. 258.

https://digitalcommons.odu.edu/bioelectrics_pubs/258

\section{Original Publication Citation}

Joshi, R. P., Hu, Q. Aly, R., Schoenbach, K. H., \& Hjalmarson, H. P. (2001). Self-consistent simulations of electroporation dynamics in biological cells subjected to ultrashort electrical pulses. Physical Review E, 64(1), 011913. doi:10.1103/PhysRevE.64.011913

This Article is brought to you for free and open access by the Frank Reidy Research Center for Bioelectrics at ODU Digital Commons. It has been accepted for inclusion in Bioelectrics Publications by an authorized administrator of ODU Digital Commons. For more information, please contact digitalcommons@odu.edu. 


\title{
Self-consistent simulations of electroporation dynamics in biological cells subjected to ultrashort electrical pulses
}

\author{
R. P. Joshi, Q. Hu, R. Aly, and K. H. Schoenbach \\ Department of Electrical and Computer Engineering, Old Dominion University, Norfolk, Virginia 23529-0246 \\ H. P. Hjalmarson \\ Computational Biology and Materials Technology Department, Sandia National Laboratory, Albuquerque, New Mexico 87185-1111 \\ (Received 6 February 2001; revised manuscript received 4 April 2001; published 21 June 2001)
}

\begin{abstract}
The temporal dynamics of electroporation of cells subjected to ultrashort voltage pulses are studied based on a coupled scheme involving the Laplace, Nernst-Plank, and Smoluchowski equations. A pore radius dependent energy barrier for ionic transport, accounts for cellular variations. It is shown that a finite time delay exists in pore formation, and leads to a transient overshoot of the transmembrane potential $V_{\text {mem }}$ beyond $1.0 \mathrm{~V}$. Pore resealing is shown to consist of an initial fast process, a $10^{-4} \mathrm{~s}$ delay, followed by a much slower closing at a time constant of about $10^{-1} \mathrm{~s}$. This establishes a time-window during which the pores are mostly open, and hence, the system is most vulnerable to destruction by a second electric pulse. The existence of such a time window for effective killing by a second pulse is amply supported by our experimental data for E. coli cells. The time constant for the longer process also matches experiments. The study suggests that controlled manipulation of the pore "open times" can be achieved through multiple, ultrashort pulses.
\end{abstract}

DOI: 10.1103/PhysRevE.64.011913 PACS number(s): 87.15.Aa, 87.50.Rr, 87.50.-a, 87.17.Aa

\section{INTRODUCTION}

Electroporation is a well-known physical process in biological cells [1-3]. It involves rapid structural rearrangement of the membrane, in response to an externally applied electric field. The most prominent observable effect is a rapid increase in the electrical conductivity by several orders of magnitude [4]. This is attributed to the formation of aqueous pathways, or pores, in the lipid bilayer of the membrane. The opening of such channels (or more appropriately, transient aqueous pores) enables the transport of ions and watersoluble species both into and out of individual cells. Electroporation can, therefore, be used to initiate large molecular fluxes for purposes of introducing genetic material into cells. Numerous related applications in molecular biology, biotechnology, and medicine are beginning to emerge [5-9].

Electroporation has also been linked to the nonthermal killing of micro-organisms subjected to strong electric fields [10]. For this reason, it offers great potential for decontamination and the elimination of harmful micro-organisms and biohazards. Traditionally, most electroporation studies have focused on relatively low external electric fields (less than a kilovolt per centimeter), applied over extended time periods ranging from several tens of microseconds to milliseconds [11]. In a very recent development, work has focused on the use of much shorter, high-voltage pulses for initiating electroporation. Electric fields as high as $100 \mathrm{kV} / \mathrm{cm}$ were used with pulse durations ranging from nanoseconds to several microseconds. There appear to be several fundamental advantages in using short electric pulses for cellular manipulation. First, negligible thermal heating of the biological matter can be expected to occur due to the short time duration. Also, much lower energies are required for pulsed inputs, and yet large values of the electric fields and peak powers can be obtained. Next, pulsed fields afford a way by which the time scales can easily be manipulated. For example, by turning off the applied fields relatively quickly, the slower processes (for example, biochemical events) could effectively be inhibited, while intracellular mechanisms would be active.

In order to fully utilize its potential of ultrashort pulses for cellular manipulation, it is important to first get a good understanding of the electroporation physics and membrane electropermeabilization. Litster [12] and Taupin, Dvolaitzky, and Sauterey [13] were the first to suggest the role of thermal fluctuations in pore formation, and the existence of a threshold pore-formation energy. The model was subsequently extended to include electrostatic energy effects [14]. Pastushenko, Chhizmadzhev, and Arakelyan [15] were the first to apply the Smoluchowski equation [16] to predict the evolutionary pore dynamics. Barnett and Weaver [17] subsequently derived the equations of Pastushenko, Chhizmadzhev, and Arakelyan [15] from statistical mechanics, and expanded the biophysical description into a numerical model [18]. Since the evolution dynamics are influenced by the transmembrane potential $U(r, t)$, calculations of $U(r, t)$ need to be included for self-consistency. Most studies, with the exception of a short report by Vaughann and Weaver [19], have ignored this aspect. Only a very recent simulation model by our group has taken account of a lumped equivalent circuit for self-consistency [20]. However, as is well known, the use of a simple lumped model has several drawbacks. First, it completely ignores the distributed nature of the internal passive circuit elements, and can lead to serious errors for high-frequency operation or on ultrashort time scales. Besides, by ignoring the spatial details, features such as the spreading resistance, which is a purely geometric effect [21], are left untreated.

The issue of charge transport during the electroporation process was studied by Chernomordik et al. [22]. A onedimensional analysis was reported by Glaser et al. [23], and subsequently generalized to three-dimensional flow by Barnett [24]. Essentially, ionic transport entails the flow of 
charged particles through an opening within a dielectric layer. Due to internal polarization, charges are induced at the membrane wall. This leads to an effective "barrier" to ionic flow that is the result of electrostatic interactions. This energy barrier can be calculated for simple geometries [25], and in general, decreases monotonically with pore radius. Due to the presence of a barrier, transport is generally weak and highly non-Ohmic at low values of the transmembrane potential, and changes dramatically as the barrier is lowered due to an external voltage. Though the barrier model appears to be adequate, more rigorous computational schemes can be applied to this problem [26]. Similarly, complex geometries for ion-flow channels could be taken into account in keeping with experimental reports. For example, besides finite cylindrical geometries [27-29], shapes such as biconical, catenary or toroidal vestibules $[30,31]$ could arguably provide a more realistic picture. In any case, the strongest contributions to the overall current will result from a relatively small subset of large pores as the corresponding energy barriers would be the least. Also, current flows can be expected to change with time as the pores expand or reseal in accordance with the Smoluchowski equation, and need to be modeled.

In this contribution, time-dependent numerical simulations have been performed to model the pore kinetics based on the governing Smoluchowski equation. Since the pore generation and resealing rates depend on the $U(r, t)$, the transmembrane potential is computed at each time step for self-consistency. Instead of using a lumped equivalent circuit approach, a coupled solution of the Laplace and current continuity equations has been used based on a three-region model for a spherical cell as described in the next section. A one-dimensional approximation of the Nernst-Planck expression has been applied for ionic transport through the pores. An energy barrier for ionic transport, taken to depend on the pore radius, accounts for cellular variations and the distributions in pore sizes. It is shown that a finite time delay exists in pore formation, and leads to a corresponding delay in the ionic current. This has two effects. First, it results in a transient overshoot of the transmembrane potential $V_{\text {mem }}$. Consequently, peak values of $V_{\text {mem }}$ can be substantially higher than the eventual steady-state level. This result agrees very well with a recent experimental report by Meier et al. [32] on giant planar lipid membranes. Second, this delay increases the input voltage magnitude necessary for causing irreversible breakdown for short electric pulses. Finally, it is shown that pore resealing is a three-step process. There is an initial fast process occurring immediately after the cessation of the external electric field. This is followed by a delay stage lasting in the $10^{-4} \mathrm{~s}$ range. Eventually, a much slower pore closing with a time constant of about $10^{-1} \mathrm{~s}$, takes over. This establishes a $0.1 \mathrm{~ms}$ time window during which the pores are mostly open, and hence, the system is most vulnerable to destruction by a second electric pulse. The existence of such a time window threshold for effective killing by a second pulse is amply supported by our experimental data. Finally, the time constant for the long-closing process also matches experimental results data and previous reports. For example, Meier et al. [32] in their experiments on giant planar membranes, also obtained a time delay of exactly $0.1 \mathrm{~ms}$.

\section{SIMULATION MODEL}

In our time-dependent simulation model of electroporation, the internal electric fields arising from an external step voltage are computed based on the current continuity and Laplace equations. Details of the electric field calculations for a spherically symmetric cell are provided later in this section. In practice, cells can be elliptical (more specifically, prolate/oblate spheroids) either due to their naturally occurring shape, or due to field related deformations. However, the creation of strong polarization at regions of sharper curvature effectively shields the electric fields, and works to minimize the disparity resulting from geometric asymmetries. Hence, the spherical geometry assumed is expected to be roughly correct. The model implicitly assumes that the time delay for cellular reorientation and alignment with the external field is negligible compared to the times scales for the poration process. Hence, the reorientation process has been ignored. This is based on an extrapolation of the characteristic delay times from the experimental data of Eynard et al. [33] to the high electrical fields of interest here. Current flows are computed corresponding to the electric fields at each time step from a continuum Nernst-Planck-type model that includes diffusion [22-24]. The transport is assumed to occur through the transient aqueous pathways (pores), and so is dictated by the time-dependent pore distribution. The requisite details regarding pore size and areal densities are computed based on the Smoluchowski equation. Details of this overall numerical model are discussed next.

In keeping with the literature [34-36], it is assumed here that two types of pores exist. The hydrophilic pores have their walls lined with water-attracting heads of lipid molecules, and are conducting. Hydrophobic pores are nonconducting, and simply represent gaps in the lipid bilayer of the membrane. Each of the two pore types are characterized by an energy of formation $E(r)$ that is a function of the pore radius $r$. In the present analysis, we have chosen to use the following pore energy function in keeping with the published and accepted model $[4,14,23,34]$ :

$$
\begin{gathered}
E(r, t)=2 \pi h r \sigma(\infty)\left[I_{1}\left(r / r_{0}\right) / I_{0}\left(r / r_{0}\right)\right]-\pi a_{\rho} V^{2} r^{2}, \underset{(1 \mathrm{a})}{\text { and }} \\
E(r, t)=2 \pi \gamma r-\left[\int_{0}^{r} 2 \pi \Gamma\left(r^{*}\right) r^{*} d r^{*}\right]+(C / r)^{2}-\pi a_{\rho} V^{2} r^{2},
\end{gathered}
$$

for hydrophobic and hydrophilic pores, respectively. In the above, $I_{1}$ and $I_{0}$ are the modified Bessel functions of the zeroth and first order, respectively, $h$ is the membrane thickness, $\sigma(\infty)$ is a constant equal to $5 \times 10^{-2} \mathrm{~N} \mathrm{~m}^{-1}$, while $r_{0}$ represents a characteristic length scale over which the properties of water change between the interface and the bulk. The value of $r_{0}$ is taken to equal $1 \mathrm{~nm}$. Also, $\gamma$ is the energy per unit length of the pore perimeter, while $\Gamma$ is the energy per unit area of the intact membrane. In practice, the $\Gamma$ value in a finite biological membrane changes with osmotic pressure, the $p \mathrm{H}$ value, and/or with perforations upon stretching. For example, a simple heuristic model has recently been used to describe such changes [37] with $\Gamma(r)=\Gamma_{0}[1$ 
TABLE I. Parameters used for the theoretical model

\begin{tabular}{lcc}
\hline \hline Parameter & Source & Value \\
\hline$D\left(\mathrm{~m}^{2} \mathrm{~s}^{-1}\right)$ & Ref. [18] & $5 \times 10^{-14}$ \\
$\gamma\left(\mathrm{J} \mathrm{m}^{-1}\right)$ & Ref. [18] & $1.8 \times 10^{-11}$ \\
$\Gamma_{0}\left(\mathrm{~J} \mathrm{~m}^{-2}\right)$ & Ref. [18] & $10^{-3}$ \\
$C\left(\mathrm{~J}^{1 / 4} \mathrm{~m}\right)$ & Ref. [23] & $9.67 \times 10^{-15}$ \\
$K_{w}\left(\mathrm{~F} \mathrm{~m}^{-1}\right)$ & Ref. [18] & $80 \times 8.85 \times 10^{-12}$ \\
$K_{m}\left(\mathrm{~F} \mathrm{~m}^{-1}\right)$ & Ref. [18] & $2 \times 8.85 \times 10^{-12}$ \\
$h(\mathrm{~m})$ & Ref. [31] & $5 \times 10^{-9}$ \\
$a_{p}\left(\mathrm{~F} \mathrm{~m}^{-2}\right)$ & Ref. [23] & $6.9 \times 10^{-2}$ \\
$v_{c}\left(\mathrm{~m}^{-3} \mathrm{~s}^{-1}\right)$ & Ref. [14] & $2 \times 10^{38}$ \\
$v_{d}\left(\mathrm{~s}^{-1}\right)$ & Ref. [31] & $10^{11}$ \\
$r_{0}(\mathrm{~m})$ & Ref. [31] & $1 \times 10^{-9}$ \\
$\left.\sigma_{0}(\mathrm{~N} \mathrm{~m})^{-1}\right)$ & Ref. [31] & $5 \times 10^{-2}$ \\
$\left.\sigma_{\text {in }}(\mathrm{S} \mathrm{m})^{-1}\right)$ & Ref. [33] & 0.455 \\
$\sigma_{\text {out }}(\mathrm{S} / \mathrm{m})$ & Ref. [33] & 5.0 \\
$\sigma(\mathrm{S} / \mathrm{m})$ & Ref. [33] & 1.3 \\
$A(\mathrm{~V})$ & Ref. [31] & 2.5 \\
$n=h_{1} / h$ & Ref. [33] & 0.15 \\
$r(\mathrm{~m})$ & Ref. [35] & $65 \times 10^{-9}$ \\
\hline \hline
\end{tabular}

$\left.-r^{2} / r_{\infty}^{2}\right]$. This is based on the idea that tension is proportional to the membrane area, at least to first order. Hence, it follows that pore formation and growth will lead to reduction in the $\Gamma$ parameter. The primary effect of such variations in $\Gamma$, would be the creation of an additional local minima in the pore energy function. From the standpoint of electroporation, this means that instead of expanding indefinitely beyond the unstable maxima, the pores become quite large, but ultimately stabilize at this high radial value. The variable tension concept had been proposed by Winterhalter and Helfrich [38], as well.

Typical values for the various parameters are given in Table I. Surface tension of the membrane, the line tension of the pore edge, and the membrane capacitance contribute to $E(r)$. The third term in Eq. (1b) represents the steric repulsion between lipid heads lining the pores $[3,35]$, and is responsible for the increase in pore free energy with shrinking radius. The value of $C$ in Eq. (1b) was chosen to be 9.67 $\times 10^{-15} \mathrm{~J}^{1 / 4} \mathrm{~m}$ in keeping with the reports by Neu and Krassowska [35] as it yields values close to those measured by Glaser et al. [23]. The last term in Eq. (1) represents the capacitive contribution to the energy in the presence of a transmembrane potential $V$. The coefficient $a_{p}$ is a property of the membrane and its aqueous environment. In the simplest continuum approximation [23], it is expressed in terms of the membrane thickness $h$ and the permittivities $\varepsilon_{w}$ and $\varepsilon_{m}$ of water and the membrane, respectively, as: $a_{p}=\left(\varepsilon_{w}\right.$ $\left.-\varepsilon_{m}\right) /[2 h]$. It might be mentioned that other models have been proposed as well that take into account different factors in the pore energy calculation. For instance, formulations based on the role of osmotic pressure [13], electrocompression of the lipid bilayer [39], interaction with membrane cytoskeleton [40], and cellular deformation [38] exist. While the expression for $E(r)$ would change somewhat on the basis of the alternative theories, the basic trends and qualitative physical behavior would remain unaltered.

The energy function $E(r)$ determines the "drift flux" for pores in $r$ space and therefore, governs the growth or contraction of pores at any given radius $r$. In general, the presence of a membrane voltage reduces the maxima, and can even quell the energy barrier completely beyond a critical voltage value. For transient voltage pulses, stability would depend on the ability of pores to drift past the barrier maximas within the duration of the applied voltage pulse. As in previous treatments, it is assumed here that the formation of pores is a two-step process. All pores, are initially created as hydrophobic/nonconducting at a rate $S(r)$ per unit area of the membrane, during every time interval $d t$. This rate is given as

$$
S(r)=\left\{\left(v_{c} h\right) /\left(k_{B} T\right)\right\}[d E(r) / d r] \exp \left[-E(r) /\left(k_{B} T\right)\right] d r d t,
$$

where $v_{c}$ is an attempt rate density [14], $E(r)$ the energy for hydrophobic pores, $T$ the operating temperature, and $k_{B}$ the Boltzmann constant. This assumes that the use of a kinetic collisional theory remains valid for nongaseous phases as well. If a nonconducting pore is created with a radius $r$ $>r^{*}(=0.5 \mathrm{~nm})$, it spontaneously changes its configuration and transforms into a conducting, hydrophilic pore. All conducting pores then survive as long as their radii remains larger than $r^{*}$. Destruction of a conducting pore occurs only if it drifts or diffuses in $r$ space to a value below $r^{*}$. Due to the exponential term in Eq. (2), most pores are created with very small radii.

The Smoluchowski equation that governs the pore dynamics is given in terms of the pore density distribution function $n(r, t)$ as

$$
\begin{gathered}
\partial n(r, t) / \partial t+\left\{D /\left[k_{B} T\right]\right\}[\partial\{E(r) n(r, t)\} / \partial r] \\
-D\left[\partial^{2} n(r, t) / \partial r^{2}\right]=S(r),
\end{gathered}
$$

where $S(r)$ is the source term as given in Eq. (2), while $D$ is the pore diffusion constant given in Table I. The process of diffusion represents a "random walk" of the pore radius in " $r$ space.' Physically, this is brought about by fluctuations in the radius in response to water molecules and other species constantly entering and leaving the pores. Numerical simulations of the dynamic pore distribution were carried out based on a time-domain, finite-difference discretization of the governing Smoluchowski equation. An upperbound $r_{\text {max }}$ of $2000 \AA$ was set on the pore radius, and this entire $r$ space was uniformly divided into 5000 segments to yield a constant grid spacing $d r$ of $0.4 \AA$. This ensured that the set limit was much larger than the critical radius $r_{c}$ at which the energy function $E(r)$ has a local maxima. Two suitable boundary conditions were imposed for the second-order partial differential equation (3). A "reflecting boundary" was assigned at $r=r_{\max }$, which was implemented by setting the pore flux to zero at $r=r_{\max }$. Mathematically, this amounts to a Neumenn condition: $\mid d n(r, t) / d r]\left.\right|_{r=r_{\text {max }}}=-[d E(r, t) / d r][n /$ $\left.\left(k_{B} T\right)\right]_{r=r_{\max }}$. At the other end, absorbing boundary conditions were implemented by setting $n(0, t)=0$. The time step 


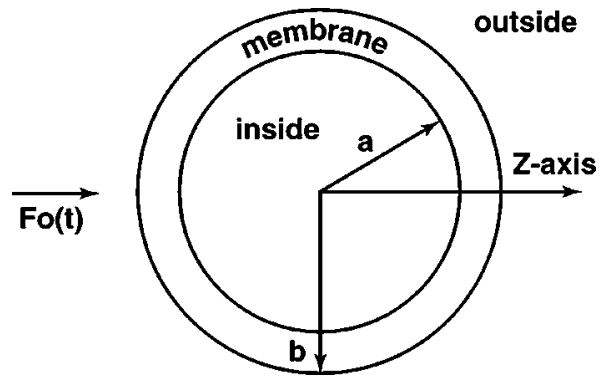

FIG. 1. Schematic of the model used to represent a cell in a suspension for potential calculations.

$d t$, in these simulations was chosen to be much smaller than the fluctuation rate $v_{d}$ that represents the fastest time constant in the system [23]. Specifically, $d t=10^{-11} \mathrm{~s}$ was used. As an initial condition prior to the application of an external voltage, the pore density was taken to be zero at all the grid points.

The external electric field $F(t)$ was taken to have the exact time-dependent shape corresponding to the external pulsed wave form. For purposes of calculating the transmembrane potential, the coupled Laplace and current-continuity equations were solved at each time step. The schematic shown in Fig. 1 was used to represent a cell suspended in a medium, and the relevant equations applied to this geometry. The geometric model is similar to that used by Grosse and Schwan [41] for analyzing membrane potentials induced by alternating fields. The inner region was assumed to be spherical in shape with a radius of $a$, homogeneous and characterized by a conductivity $\sigma_{\text {in }}$. The applied electric field $F_{0}(t)$ was taken to be along the $z$ axis. The cellular membrane was assigned a thickness $b$ - $a$, while the outer region denoting the suspension was assigned a conductivity $\sigma_{\text {out }}$. Due to spherical symmetry, the potentials that must satisfy the Laplace equation, can be expressed as

$$
\begin{aligned}
& U_{\text {in }}(r, t)=A_{0}(t) P_{0}+A_{1}(t) r P_{1}+A_{2}(t) r^{2} P_{2}+\cdots \\
& =\sum_{j=0, \infty} A_{j}(t) r^{j} P_{j} \\
& U_{\mathrm{mem}}(r, t)=\sum_{j=0, \propto}\left[B_{j}(t) r^{j} P_{j}+C_{j}(t) P_{j} / r^{j+1}\right], \\
& \text { and } U_{\text {out }}(r, t)=-F_{0}(t) r P_{1}+\sum_{j=0, \propto} D_{j}(t) P_{j} / r^{j+1} \text {, }
\end{aligned}
$$

where $U_{\text {in }}(r, t), U_{\text {mem }}(r, t)$, and $U_{\text {out }}(r, t)$ are the potentials at the inner, the membrane, and outer regions, $P_{j}$ is the $j$ th order Legendre polynomial, and $F_{0}(t)$ the externally applied electric field. Also, $A_{j}(t), B_{j}(t), C_{j}(t)$, and $D_{j}(t)$ are the coefficients of the Legendre series expansions that can be determined by applying matching boundary conditions at the interfaces of the three regions. Here, the Laplace instead of Poisson's equation has been used on the assumption that charge inequalities arsing from ionic transport during the electroporation process can be ignored on the short time scales. As will be shown later, the current flows are not very large and so charge transfer during the ultrashort time scales of interest here, are indeed minimal. Invoking continuity in the potential and current density then leads to the following boundary conditions:

$$
\begin{gathered}
U_{\text {in }}(r=a, t)=U_{\text {mem }}(r=a, t), \\
U_{\text {mem }}(r=b, t)=U_{\text {out }}(r=b, t), \\
\left.\sigma_{\text {in }}\left[\partial U_{\text {in }}(r, t) / \partial r\right]\right|_{r=a}=\left.\sigma_{\text {out }}\left[\partial U_{\text {out }}(r, t) / \partial r\right]\right|_{r=b}, \quad(5 \mathrm{c}) \\
\text { and }-\left.\sigma_{\text {in }}\left[\partial U_{\text {in }}(r, t) / \partial r\right]\right|_{r=a}=C_{M}\left[\partial V_{\text {mem }}(t) / \partial t\right]+J_{\mathrm{mem}}(t),
\end{gathered}
$$

where $C_{M}$ is the membrane capacitance, $V_{\text {mem }}(t)=\left[U_{\text {mem }}(r\right.$ $\left.=b, t)-U_{\text {mem }}(r=a, t)\right]$ is the membrane potential, and $J_{\text {mem }}(t)$ is the conduction current density across the membrane through the pores. Values of the conductivity parameters and the membrane capacitance have been reported in the literature [42], and are used here as given in Table I. Straightforward, but tedious manipulation of Eq. (5) yields the following expression for the time-dependent membrane potential $V_{\text {mem }}(t)$ :

$$
\begin{aligned}
V_{\text {mem }}(t)= & A(t) \cos (\theta)\left[\left\{b-b^{3} / a^{2}\right\}\left\{1+2 \sigma_{\text {out }} / \sigma_{\text {in }}\right\} /\left\{(b / a)^{3}\right.\right. \\
& \left.\left.+2 \sigma_{\text {out }} / \sigma_{\text {in }}\right\}-(b-1)\right]+3 \cos (\theta) F_{0}(t) \\
& \times\left(\sigma_{\text {out }} / \sigma_{\text {in }}\right) /\left\{(b / a)^{3}+2 \sigma_{\text {out }} / \sigma_{\text {in }}\right\}
\end{aligned}
$$

where $\theta$ is the angle with respect to the $z$ axis (and hence, the applied electric field direction), and $A(t)$ satisfies the following ordinary differential equation:

$$
\begin{aligned}
C_{M}[ & d A(t) / d t]\left[\left\{b-b^{3} / a^{2}\right\}\left\{1+2 \sigma_{\text {out }} / \sigma_{\text {in }}\right\} /\left\{(b / a)^{3}\right.\right. \\
+ & \left.\left.2 \sigma_{\text {out }} / \sigma_{\text {in }}\right\}-(b-a)\right] \\
= & -J_{\text {mem }}(t)-\sigma_{\text {in }}\left(\left[2\left(\sigma_{\text {out }} / \sigma_{\text {in }}\right)\left\{(b / a)^{3}-1\right\} A(t)\right.\right. \\
& \left.-3\left(\sigma_{\text {out }} / \sigma_{\text {in }}\right) F_{0}(t)(b / a)^{3}\right] /\left\{(b / a)^{3}\right. \\
& \left.\left.+2 \sigma_{\text {out }} / \sigma_{\text {in }}\right\}\right) .
\end{aligned}
$$

Since steady-state results of the transmembrane potential have been derived in the literature, an indirect validation of the above equation can easily be obtained by evaluating $V_{\text {mem }}(t)$ in the long time limit. Setting the time derivative term to zero yields an expression for $A(t \rightarrow \propto)$. Using this expression in equation (6a), provides the steady-state transmembrane potential: $V_{\text {mem }}=-1.5 a E \cos (\theta)$ for $b \sim a$. This steady-state result is in accordance with the expression reported and experimentally verified in the literature $[43,44]$. It is worth mentioning that the time-varying field of equation (4), would produce a force at the membrane in accordance with the Maxwell stress tensor [45]. It is conceivable that such stress will lead to changes in the volume and shape of the cells. Experimental scattering data on cells subjected to high-voltage pulses confirms such dynamical variations in 
size [46]. Cell shrinkage in the context of apoptosis following high-voltage pulses is also possible. Such aspects can be analyzed based on the theory presented here. However, numerical results and pertinent discussions on volumetric changes will be reported elsewhere.
The conduction current density $J_{\text {mem }}(t)$ needs to be specified in order to solve for the potentials in Eq. (6). A onedimensional approximation of the Nernst-Planck expression for ionic flow has often been used in the literature [22-24]. This gives to the following current-voltage relation:

$$
I(t)=\pi \sigma R^{2} N(t)\left\{\exp \left[q V_{\mathrm{mem}}(t) /\left(k_{B} T\right)\right]-1\right\} /\left(\int_{0}^{h} \exp \left[q V_{\mathrm{mem}}(t)\{1-x / h\} /\left(k_{B} T\right)+w(x)\right] d x\right),
$$

where $R(t)$ is the pore radius, $\sigma$ the conductivity of the aqueous solution that fills the pore, $w(x)$ the energy barrier to ionic flow through the pores, $h$ is the membrane thickness, and $N(t)$ the pore density. A simple trapezoidal form for $w(x)$ as given by

$$
\begin{gathered}
w(x)=q A /\left(k_{B} T\right)\left\{x / h_{1}\right\} \quad \text { for } 0 \leqslant x \leqslant h_{1} . \\
w(x)=q A /\left(k_{B} T\right) \text { for } h_{1} \leqslant x \leqslant h-h_{1} . \\
w(x)=q A /\left(k_{B} T\right)\left\{(h-x) / h_{1}\right\} \quad \text { for } h_{1} \leqslant x \leqslant h-h_{1} .
\end{gathered}
$$

has been used for the barrier energy, and will be applied here. In the above, $A$ represents the peak barrier height under zero bias. Values of $\sigma$ and $A$ are known to be roughly 1.3 $\mathrm{S} / \mathrm{m}$ [42] and $2.5 \mathrm{~V}$ [23], respectively. The parameter $h_{1}$ is the length of the entrance region of the pore over which the barrier profile would be changing linearly for an unbiased cell. Its value is roughly 0.15 times the membrane thickness. In addition, an ionic component, $I_{\mathrm{ion}}$, which is orders of magnitude lower in strength, has to be included. This ionic current density is [42]:

$$
I_{\text {ion }}=1.9\left(V_{\text {mem }}+0.083\right) .
$$

The $83 \mathrm{mV}$ in the equation above represents the reversal potential.
It must be pointed out that the above equations (7) and (8) are somewhat inaccurate for the following reasons.

(i) First, the barrier peak $A$ is assumed to be independent of the pore radius. This is physically inaccurate. As shown by Parsegian [25], for example, the barrier is a monotonically decreasing function of the radius $r$ implying that it is easier for ions to get through wider pores. An approximate form, which is correct for an infinitely long cylindrical pore geometry, for $w(r)$ has been derived to be [25]: $w(r)=5$ $\times 10^{-9} / r$. In our calculations, this $w(r)$ function was explicitly used in Eq. (8).

(ii) Next, Eq. (7) treats the pore radius to be a constant that is incorrect for two reasons. Not only would the radius of the pores change under transient conditions upon the application of an external voltage wave form, but also the pores would not all be identical in size. The distribution $n(r, t)$ as predicted by the Smoluchowski equation, would impart a heterogeneous spread in the $R$ parameter.

(iii) Finally, $N(t)$ that is an integral quantity needs to be obtained through a suitable integration of $n(r, t)$ over $r$ space. Treating $N(t)$ as a fundamental independent variable is incorrect.

To redress the above shortcomings of equation (7), a somewhat modified current-voltage $(I-V)$ characteristics were used in this study. The $I-V$ relation was taken to be

$$
I(t)=\pi \sigma \int_{0}^{\infty}\left(r^{2} n(r, t)\left\{\exp \left[q V_{\mathrm{mem}}(t) /\left(k_{B} T\right)\right]-1\right\} /\left\{\int_{0}^{h} \exp \left[q V_{\mathrm{mem}}(t)\{1-x / h\} /\left(k_{B} T\right)+w(r, x)\right] d x\right\}\right)
$$

which includes an integration in $r$ space over the timedependent distribution $n(r, t)$. In the process, the role of intercellular variations and the size distribution of pores are both taken into account. A distribution of barrier energies and their fluctuation from site to site is also automatically included in this formulation. It, therefore, represents a more physical model of an actual cellular colony.

As a final comment, it may be pointed out that the present model is non-Markovian in nature, and hence, includes memory effects. The kinetic rates depend on the membrane voltage, and hence, vary with time. Consequently, the model describing the evolution of the biological system, not only depends on the initial starting state, but also on the details of the time-dependent voltage sequence. This formulation, therefore, goes beyond the Markovian treatments based on Poisson models $[47,48]$.

\section{RESULTS AND DISCUSSION}

The pore formation energy function for hydrophilic pores is shown in Fig. 2 based on Eq. (1b) for various values of the $r_{\propto}$ parameter. Its characteristics are important since the pore 


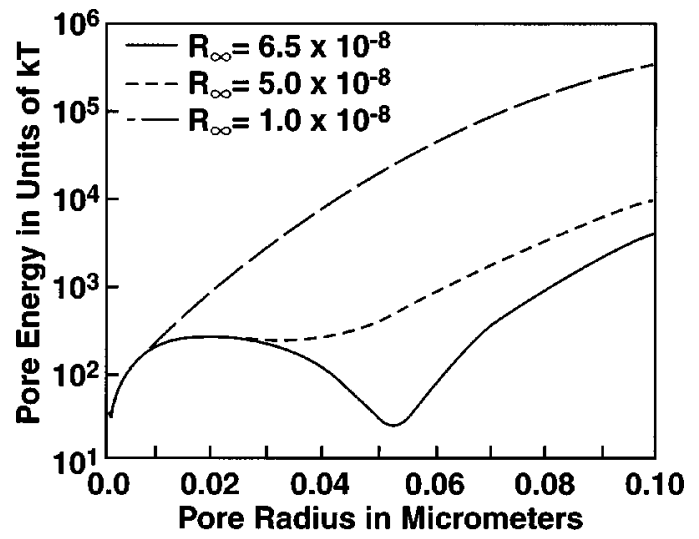

FIG. 2. The pore formation energy function for hydrophilic pores for various $r_{\infty}$ values.

dynamics are governed by this energy function. Essentially energy is required to create a "circular" edge for pore formation. However, a deduction has to be considered to account for the elimination of the membrane surface area. For a monotonically decreasing value of the surface tension with pore radius, as implied by a finite $r_{\propto}$ parameter, contributions to an energy decrease are reduced leading to increases in the formation energy. This is evident from the three curves of Fig. 2 corresponding to three different $r_{\propto}$ values. A fixed, high value of $\Gamma$ corresponding to the $r_{\propto}$ $=\propto$ limit yields the lowest energy. Physically though, $r_{\propto}=$ $\propto$ is an incorrect representation for two reasons. First, it implies that the pores are able to expand without bound and never stabilize. This would lead to unphysical density enhancements in the nonporous regions of the membrane, or thickness increases. Second, $r_{\alpha}=\propto$ incorrectly implies that the tension is unaffected by changes in the membrane area. Though direct experimental verification of surface tension is unavailable, molecular dynamics simulations of lipid bilayers do demonstrate the following [49]: (a) Finite tension is required to maintain a given cellular shape and size, and (b) the tension must change with the system area. Also, indirect experimental evidence indicative of variations in membrane tension is available. For example, activation of the 3-ns mechanosensitive channel large cloned from $E$. coli $[50]$ has been linked to the tension of lipid membranes. Similarly, the activity of lytic peptides is affected by the tension of vesicles under stress [51], and the catalytic activity of a $\beta$ isoform of phospholipase $C$ shown to change with surface pressure [52]. These experimental results suggest that the tension must naturally be variable, and that its variation facilitates biological activities that are observed. Third, since tension is proportional to the membrane area, at least to first order, it follows that changes due to pore formation will lead to variations in $\Gamma$ that are proportional to the square of the pore radius.

For a finite value of $r_{\propto}$, the formation energy not only increases, but also exhibits a local minima. This implies that the pores can expand upon the application of external electric fields, but will eventually stabilize to some large average value as dictated by the minima. This leads to the following consequences. (i) First, for pore creation and cellular ma- nipulation, a somewhat larger external energy will be necessary to create large pores due to the variable surface tension. (ii) Second, for cellular destruction, a sufficiently large voltage must be applied for a sufficiently long time to transcend the energy barrier and ensure that pores move into the energy minima. (iii) Pore resealing, in the presence of a local minima (e.g., as in the $r_{\propto}=65 \mathrm{~nm}$ curve of Fig. 2), becomes a two-step process. An initial rapid decrease is expected to occur due to the diffusion of pore with radii smaller than the local minima toward small $r$ space. However, those lying beyond the local minima will remain "trapped" and will diffuse to lower $r$ values very slowly. Hence, conceivably, a small fraction of the pore will remain open for long times, provided the initial voltage and time duration were sufficient to carry them over the barrier. (iv) Finally, the two-step process implies that there is an optimal time-window for cellular destruction. If, for example, a second voltage pulse can be applied before the fast first-step has not fully completed, the potential for damage will be rather high. On the other hand, applying a second voltage pulse after the end of the fast resealing process, only a few pores will be open, and a high membrane potential will not be developed due to their high conductivity. This suggests that for maximal damage, a series of short pulses with delays less than that of the fastprocess time constant will be most desirable.

Self-consistent simulations based on the coupled LaplaceNernst-Planck-Smoluchowski equations were carried out next to evaluate the temporal response to ultrashort, external electric pulses. A $10 \mathrm{kV} / \mathrm{cm}$ rectangular external electric field pulse with a $4 \times 10^{-6}$ duration was assumed. These parameters were chosen in keeping with actual pulsed field experiments conducted on E. coli in our laboratory to facilitate comparisons between theory and experiment. The cell radius was chosen to be $1.0 \mu \mathrm{m}$ and a membrane thickness of $5 \mathrm{~nm}$ which is roughly characteristic of E. coli. Figure 3 shows the dynamic evolution of the pore density. An initial delay of about 5 ns seen in Fig. 3(a) is due to membrane charging and for $V_{\text {mem }}$ to build up to levels at which the pore creation rate becomes substantial. A peak value of about $2 \times 10^{14} \mathrm{~m}^{-2}$ is reached after about 20 ns. Subsequently, the pore density shows a slight monotonic decrease over the remaining duration of the $4 \mu$ s external pulse. This occurs due to a substantial increase in the membrane conductance and a consequent decay in $V_{\text {mem }}$ that controls the pore generation. Details of the time-dependent membrane voltage are shown in Fig. 4. The voltage exceeds $1.0 \mathrm{~V}$ at about $15 \mathrm{~ns}$, and reaches a peak value of roughly $1.2 \mathrm{~V}$. At this point the pore conductance increases to such a degree that the voltage across the membrane capacitance begins to fall. The overall result is a "voltage overshoot" behavior. It agrees well with a previous report on the time-dependent behavior of the membrane voltage by Meier et al. [32]. As the external electric field is turned off beyond $4 \mu \mathrm{s}$, the transmembrane potential falls dramatically with a time constant in the sub-microsecond range. The fast dropoff is the result of a large conductance, and hence, a low internal "RC" time constant. A final steady-state value of about $-80 \mathrm{mV}$, equal to the rest potential is finally reached. The corresponding influence on the pore density, as seen from Fig. 3(a), is a sharp decrease by 

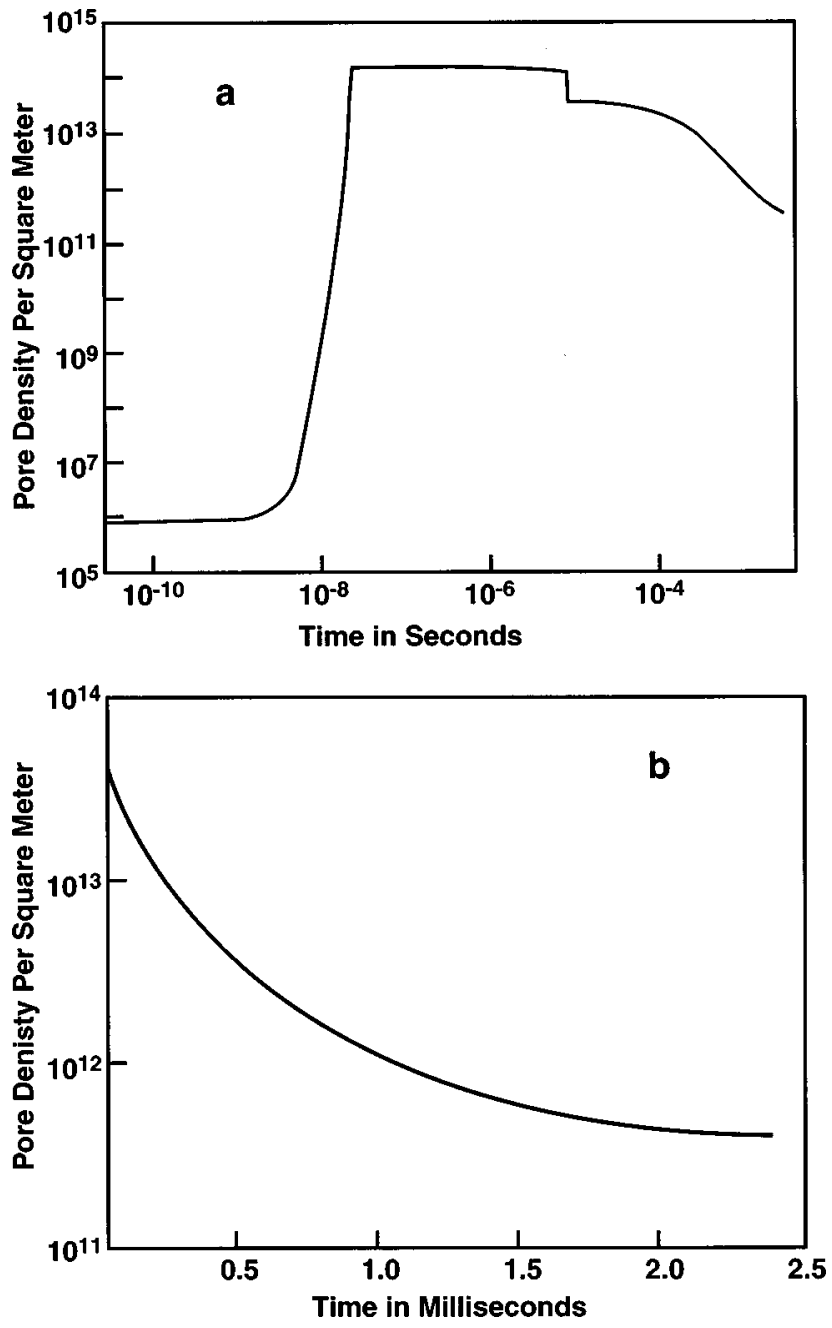

FIG. 3. Simulation result for the pore density evolution with time in response to a $4 \mu \mathrm{s}, 10 \mathrm{kV} / \mathrm{cm}$ rectangular electric pulse. (a) Logarithmic scale, and (b) semilogarithmic graph.

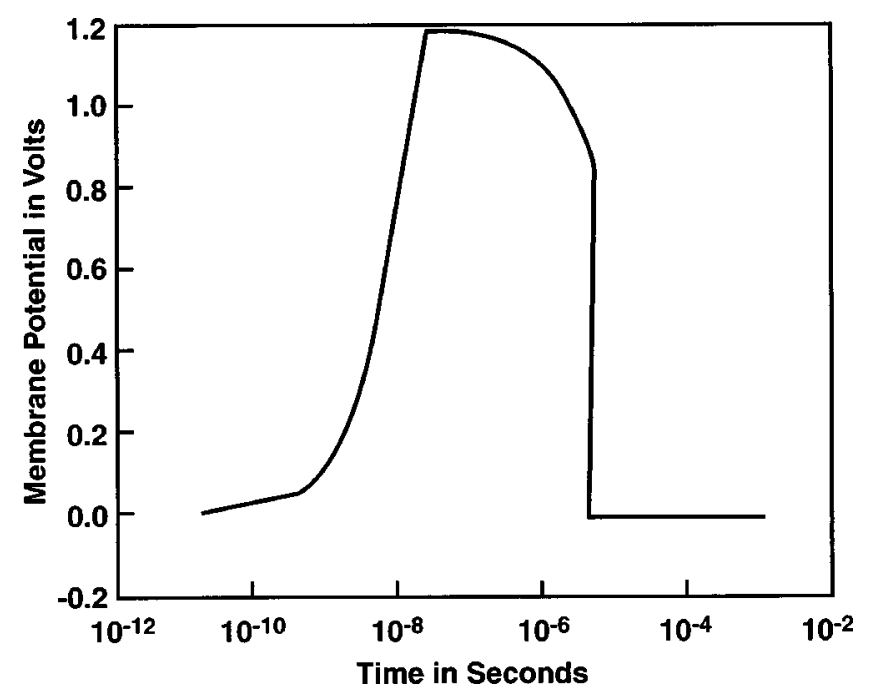

FIG. 4. Calculated temporal variations of the transmembrane potential in response to a $4 \mu \mathrm{s}, 10 \mathrm{kV} / \mathrm{cm}$ rectangular electric pulse.

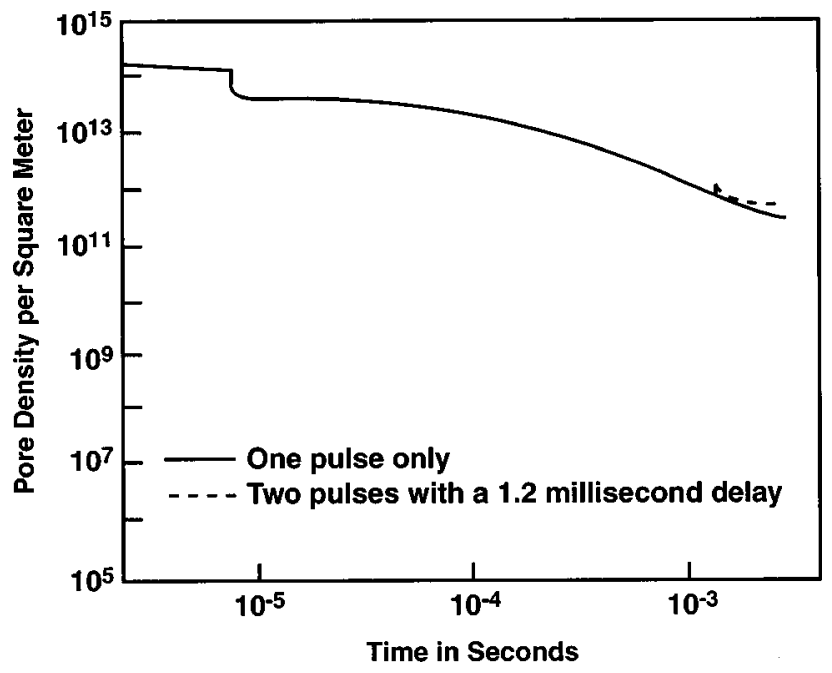

FIG. 5. Simulation result comparing the pore density evolution with one and two $4 \mu \mathrm{s}, 10 \mathrm{kV} / \mathrm{cm}$ rectangular electric pulses. The delay for the two-pulse simulation was taken to be $1.2 \mathrm{~ms}$.

about fifty percent following the turn-off. Beyond this, the density continues to decrease, but at a relatively low rate until about $0.1 \mathrm{~ms}$. This implies that many of the pores tend to remain open, well after the $4 \mu$ s pulse is switched off. Hence, a second electric pulse applied within this duration is likely to have a substantial damaging effect. This is borne out in our experimental measurements on $E$. coli, as discussed later. Also, this $0.1 \mathrm{~ms}$ time delay corresponds well with an experimental report by Meier et al. [32]. Beyond 0.1 $\mathrm{ms}$, the rate of pore reduction increases. The long time behavior can best be gauged from the semilogarithmic curve of Fig. 3(b). It shows a gradual slowing in the resealing rate. At the $2.4 \mathrm{~ms}$ instant, an "effective decay time constant" of $4.5 \times 10^{-3} \mathrm{~s}$ is computed from the results. Based on this time constant, a lower bound on the duration for near-complete pore resealing can be estimated. It works out to $0.12 \mathrm{~s}$. In actuality, though, the duration would be even longer since the decay curve of Fig. 3(b) exhibits a continuous slowing down. It is, therefore, natural to expect that the pore decay will weaken even further at longer times, and resealing durations spanning several seconds or even minutes, will result. In any case, the projected resealing values are in the $10^{-1}-10^{2} \mathrm{~s}$ range, in keeping with experimental reports $[53,54]$.

The dynamical behavior can easily be understood in terms of the formation energy characteristic of Fig. 2. Initially, the pores that are not near the local minima, and have values in $r$ space that are below the barrier. These pores tend to drift and diffuse toward $r \rightarrow 0$, giving rise to a fast decay. However, this leaves behind an ever-increasing higher fraction of larger pores that are near the local minima. Those near the minima move in $r$ space primarily through diffusion, and hence, the system takes a long time to completely recover to the original steady state.

The effect of including a second electric pulse and the comparative temporal behavior is shown in the curves of Fig. 5 . The pore density evolution for single and a dual $4 \mu \mathrm{s}, 10$ $\mathrm{kV} / \mathrm{cm}$ rectangular electric pulses is shown. The delay for the 


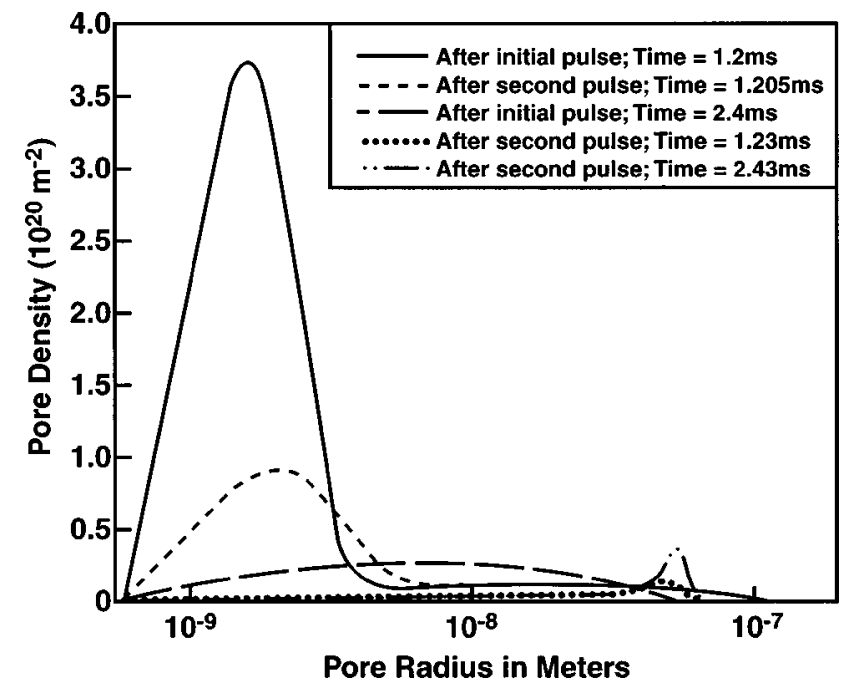

FIG. 6. Simulated pore distribution functions at various times for single- and dual-pulse situations.

two-pulse situation was taken to be $1.2 \mathrm{~ms}$. Despite being outside the $0.1 \mathrm{~ms}$ time-window, the application of a second pulse is seen to have effect on the evolution. First, the pore density is increased leading to a short spike. However, this increment is not as large as that produced by the original pulse, since the larger pores remain open, giving rise to a reasonably large membrane conductivity. Consequently, the membrane potential developed is not as large, and the pore creation rate not boosted as much. A second observation is that a two-step decay is evident following the second pulse, as with the initial pulse. Finally, and most important, the decay time for the two-pulse situation becomes significantly longer beyond $2 \mathrm{~ms}$. This implies that applying two or more pulses can slow resealing considerably. From a practical standpoint, this has applications for drug delivery over prolonged durations. The resealing rate reduction for a dualpulse scenario can again be understood on the basis of the formation energy curve. The second pulse forces more pores over the energy barrier and into the local minima. With an increased fraction of such pores, the decay rate is correspondingly reduced.

For a more quantitative evaluation, it is instructive to examine the pore distribution functions at various times. Shown in Fig. 6 are five distribution functions at various times for the single- and dual-pulse scenarios. The highest curve for the dual-pulse situation is at a time instant of 1.205 $\mathrm{ms}$ and hence, just after the completion of the second pulse. Though the peak lies at about $1.5 \mathrm{~nm}$, a fairly broad "tail" at larger radii is apparent. At a later time of $t=1.23 \mathrm{~ms}$ for the dual-pulse situation, the peak is seen to be reduced appreciably and broadened due to diffusion in $r$ space. Finally, at the longest time of $2.43 \mathrm{~ms}$, the dual-pulse distribution is seen to have become quite negligible for small pore radii, but has a well-defined peak at about $52 \mathrm{~nm}$. This location corresponds to the potential minima in Fig. 2 for the $r_{\alpha}=65 \mathrm{~nm}$ curve. It is thus evident that a well-defined population of relatively large pores remains and is long-lived. For the single-pulse situation, the snapshot distributions at $t=1.2$ and $2.4 \mathrm{~ms}$ are shown. The distribution is relatively broad at $1.2 \mathrm{~ms}$, and

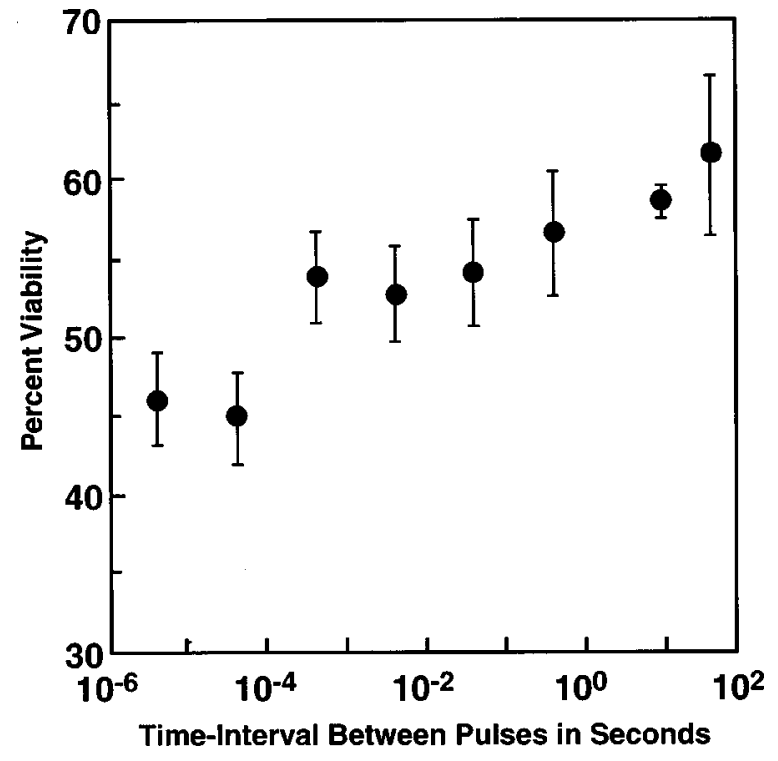

FIG. 7. Experimental results on the viability of E. coli subjected to two $4 \mu \mathrm{s}, 13 \mathrm{kV} / \mathrm{cm}$ electric pulses with variable delays. The delay between the first and second pulses ranged from $5 \mu$ s to $50 \mathrm{~s}$.

similar to the dual-pulse case, settles down to acquire a local peak at $52 \mathrm{~nm}$. However, the $52 \mathrm{~nm}$ peak is smaller, signifying a greater number of pores for the dual-pulse case. Beyond these times, the resealing can be expected to be slow, and pore may remain open for a very long time.

Finally, some experimental findings and measured data for $E$. coli cells subjected to short electric pulses are presented. The results, plotted in Fig. 7, show the measured viability of $E$. coli subjected to two $4 \mu \mathrm{s}, 13 \mathrm{kV} / \mathrm{cm}$ electric pulses with variable delays. The delay between the first and second pulses ranged from $5 \mu$ s to $50 \mathrm{~s}$. A sudden jump in the viability is seen in going from a $0.1 \mathrm{~ms}$ delay to a $1.0 \mathrm{~ms}$ delay for the second pulse. This is in keeping with the simulation result of Fig. 3(a) that shows a $0.1 \mathrm{~ms}$ time-window during which the pores are mostly open, making the system most vulnerable to destruction by a second electric pulse. Second, there is no significant change in the experimental viability in going from a $1.0 \mathrm{~ms}$ delay to a $1 \mathrm{~s}$ delay. This again is in keeping with the prediction of a slow pore decay, and the calculated lower bound of about $0.12 \mathrm{~s}$. Finally, the absence of complete saturation in the viability even for delay times as long as $50 \mathrm{~s}$, suggests that the pores are relatively long lived in accordance with previous reports $[53,54]$.

\section{SUMMARY AND CONCLUSIONS}

A self-consistent model analysis of electroporation in biological cells has been carried out based on the coupled Laplace-Nernst-Planck-Smoluchowski equations. The physical processes of pore generation, drift, and diffusion in $r$ space were all comprehensively included. A pore-radiusdependent energy barrier to ionic transport accounted for cellular variations. The primary objective was to obtain predictions and qualitative understanding of the cellular response to short, high electric field pulses by taking account of the growth and resealing dynamics. The electroporation dynam- 
ics in the presence of multiple electric pulses and the potential benefits have also been analyzed.

It has been shown that a finite time delay exists in pore formation, and leads to a transient overshoot of the transmembrane potential $V_{\text {mem }}$. The membrane potential itself remains around the $1.0 \mathrm{~V}$ value that has been reported in the literature. However, the peak can exceed this value on a transient basis. It has also been demonstrated that pore resealing is a multistep process. It consists of an initial fast decay, followed by a $10^{-4} \mathrm{~s}$ delay, and then a much slower poreclosing with a time constant of about $10^{-1} \mathrm{~s}$. This establishes a 0.1 (ms) time-window during which the pores are mostly open, and hence, the system is most vulnerable to destruction by a second electric pulse. The existence of such a time window threshold for effective killing by a second pulse is amply supported by our experimental data for E. coli cells involving two pulses with variable delays. A sudden jump in the experimental cell viability has been observed in going from a $0.1 \mathrm{~ms}$ delay to a $1.0 \mathrm{~ms}$ delay for the second pulse. This is in keeping with our simulation results that show a 0.1 ms time window during which the pores are mostly open.
Second, no significant change in the experimental viability was recorded in going from a $1.0 \mathrm{~ms}$ delay to a $1 \mathrm{~s}$ delay. This again is in keeping with the prediction of slow pore decay, and the calculated lower bound of about $0.12 \mathrm{~s}$. The time constant for the longer process also matches experimental results and previous reports.

Finally, it has also been shown that the pore decay time for the two-pulse situation becomes significantly longer than a single pulse case. This implies that applying two or more pulses can slow resealing considerably. From a practical standpoint, this has applications for drug delivery over prolonged durations, or for controlled manipulation of the pore "open times" via ultrashort, multiple pulses.

\section{ACKNOWLEDGMENTS}

The authors would like to thank J. Weaver (MIT) and W. Krassowska (Duke University) for useful discussions. This work was sponsored in part by the Air Force Office of Scientific Research. Support from Old Dominion University is also gratefully acknowledged.
[1] R. Stampfli, Z. Chem. 30, 57 (1958).

[2] T. Y. Tsong, Biophys. J. 60, 297 (1991).

[3] J. C. Weaver and Yu. A. Chizmadzhev, Bioelectrochem. Bioenerg. 41, 135 (1996).

[4] I. G. Abidor, V. B. Arakelyan, L. V. Chernomordik, Y. A. Chizmadzhev, V. F. Pastushenko, and M. R. Tarasevich, Bioelectrochem. Bioenerg. 6, 37 (1979).

[5] G. A. Hoffman, S. B. Dev, and G. S. Nanda, IEEE Trans. Biomed. Eng. 46, 752 (1999).

[6] D. C. Chang, B. M. Chassy, J. A. Saunders, and A. E. Sowers, Guide to Electroporation and Electrofusion (Academic, New York, 1992).

[7] E. Neumann, E. Sowers, and C. A. Jordan, in Electroporation and Electrofusion in Cell Biology, edited by E. Neumann, E. Sowers, and C. A. Jordan (Plenum, New York, 1989).

[8] S. Orlowski and L. M. Mir, Biochim. Biophys. Acta 1154, 51 (1993).

[9] J. C. Weaver, J. Cell. Biochem. 51, 426 (1993).

[10] A. J. H. Sale and W. A. Hamilton, Biochim. Biophys. Acta 148, 781 (1967).

[11] H. Huelsheger, J. Potel, and E. G. Niemann, Radiat. Environ. Biophys. 20, 53 (1981).

[12] J. D. Litster, Phys. Lett. 53A, 193 (1975).

[13] C. Taupin, M. Dvolaitzky, and C. Sauterey, Biochim 14A, 4771 (1975).

[14] J. C. Weaver and R. A. Mintzer, Phys. Lett. 86A, 57 (1981).

[15] V. F. Pastushenko, Yu A. Chhizmadzhev, and V. B. Arakelyan, Bioelectrochem. Bioenerg. 6, 53 (1979).

[16] For example, R. K. Pathria, Statistical Mechanics (Pergamon, Oxford, 1972), pp. 451-455.

[17] A. Barnett and J. C. Weaver, Bioelectrochem. Bioenerg. 25, 163 (1991).

[18] S. A. Freeman, M. A. Wang, and J. C. Weaver, Biophys. J. 67, 42 (1994).
[19] T. Vaughan and J. C. Weaver, in Electricity and Magnetism in Biology and Medicine, edited by A. Bersani (Kluwer Academic, New York, 1999), p. 433.

[20] R. P. Joshi and K. H. Schoenbach, Phys. Rev. E 62, 1025 (2000).

[21] R. Holm, Electric Contacts Theory and Applications (Springer-Verlag, Berlin, 1967).

[22] L. V. Chernomordik, S. I. Sukharev, S. V. Popov, V. F. Pastushenko, A. I. Sokirko, I. G. Abidor, and Y. A. Chizmadzhev, Biochim. Biophys. Acta 902, 360 (1987).

[23] R. W. Glaser, S. L. Leikin, L. V. Chernomordik, V. F. Pastushenko, and A. I. Sokirko, Biochim. Biophys. Acta 940, 275 (1988).

[24] A. Barnett, Biochim. Biophys. Acta 1025, 10 (1990).

[25] A. Parsegian, Nature (London) 221, 844 (1969).

[26] L. J. Douglas Frink and F. van Swol, J. Chem. Phys. 106, 3782 (1997).

[27] P. C. Jordan, Biophys. J. 39, 157 (1982); P. C. Jordan, ibid. 41, 189 (1983).

[28] S. Bek and E. Jakobsson, Biophys. J. 66, 1028 (1994).

[29] S. W. Chiu and E. Jakobsson, Biophys. J. 55, 1028 (1989).

[30] S. C. Li, M. Hoyles, S. Kuyucak, and S. H. Chung, Biophys. J. 74, 37 (1998).

[31] M. Hoyles, S. Kuyucak, and S. H. Chung, Biophys. J. 70, 1628 (1996).

[32] W. Meier, A. Graff, A. Diederich, and M. Winterhalter, Phys. Chem. Chem. Phys. 2, 4559 (2000).

[33] N. Eynard, F. Rodriguez, J. Trotard, and J. Teissie, Biophys. J. 75, 2587 (1998).

[34] J. C. Weaver, Ann. Acad. Sci. 720, 141 (1994).

[35] J. C. Neu and W. Krassowska, Phys. Rev. E 59, 3471 (1999).

[36] J. Teissie and M. P. Rols, Ann. Acad. Sci. 720, 98 (1994).

[37] H. Isambert, Phys. Rev. Lett. 80, 3404 (1998).

[38] M. Winterhalter and W. Helfrich, Phys. Rev. A 36, 5874 (1987). 
[39] I. P. Sugar, Biochim. Biophys. Acta 556, 72 (1979).

[40] W. Sung and P. J. Park, Biophys. J. 73, 1797 (1997).

[41] C. Grosse and H. P. Schwan, Biophys. J. 63, 1632 (1992).

[42] K. A. DeBruin and W. Krassowska, Biophys. J. 77, 1213 (1999).

[43] D. Gross, L. M. Loew, and W. W. Webb, Biophys. J. 50, 339 (1986).

[44] Z. Lojewska, D. L. Farkas, B. Ehrenberg, and L. M. Loew, Biophys. J. 56, 121 (1989).

[45] For example, J. D. Jackson, Classical Electrodynamics (Wiley, New York, 1962).

[46] E. Neumann, S. Kakorin, and K. Toensing, Faraday Discuss. 111, 111 (1998).
[47] I. P. Sugar, in Electroporation and Electrofusion in Cell Biology (Ref. [7]), pp. 97-110.

[48] G. Saulis, Biophys. J. 73, 1299 (1997).

[49] S. E. Feller and R. W. Pastor, Biophys. J. 71, 1350 (1996).

[50] S. I. Sukharev, P. Blount, B. Martinac, F. R. Blattner, and C. Kung, Nature (London) 368, 265 (1994).

[51] T. Benachir and M. Lafleur, Biophys. J. 70, 831 (1996).

[52] S. R. James, R. A. Demel, and C. P. Downes, Biophys. J. 298, 499 (1994).

[53] D. C. Chang and T. S. Reese, Biophys. J. 58, 1 (1990).

[54] I. Tsoneva, T. Tomov, L. Panova, and D. Strahilov, Bioelectrochem. Bioenerg. 24, 41 (1990). 